\title{
Staging FDG PET-CT changes management in patients with gastric adenocarcinoma who are eligible for radical treatment
}

\author{
Karen D. Bosch ${ }^{1}$ (D) Sugama Chicklore ${ }^{2,3} \cdot$ Gary J. Cook $^{2,3} \cdot$ Andrew R. Davies ${ }^{1} \cdot$ Mark Kelly $^{1} \cdot$ James A. Gossage ${ }^{1} \cdot$ \\ Cara R. Baker ${ }^{1}$
}

Received: 31 January 2019 / Accepted: 9 July 2019 / Published online: 3 August 2019

(C) The Author(s) 2019

\begin{abstract}
Aim 18-fluorodeoxyglucose positron emission tomography-computed tomography (FDG PET-CT) is valuable in the management of patients with oesophageal cancer, but a role in gastric cancer staging is debated. Our aim was to review the role of FDG PET-CT in a large gastric cancer cohort in a tertiary UK centre.

Methods We retrospectively reviewed data from 330 patients presenting with gastric adenocarcinoma between March 2014 and December 2016 of whom 105 underwent pre-treatment staging FDG PET-CT scans. FDG PET-CT scans were graded qualitatively and quantitatively $\left(\mathrm{SUV}_{\max }\right)$ and compared with staging diagnostic $\mathrm{CT}$ and operative pathology results $(n=30)$ in those undergoing resection.

Results Of the 105 patients ( $74 \mathrm{M}$, median age 73 years) $86 \%$ of primary tumours were metabolically active (uptake greater than normal stomach) on FDG PET-CT [41/44 (93\%) of the intestinal histological subtype (SUV $\max 14.1 \pm 1.3$ ) compared to 36/46 (78\%) of non-intestinal types ( $\left.\left.\mathrm{SUV}_{\max } 9.0 \pm 0.9\right), p=0.005\right]$. FDG PET-CT upstaged nodal or metastastic staging of 20 patients (19\%; 13 intestinal, 6 non-intestinal, 1 not reported), with 17 showing distant metastases not evident on other imaging. On histological analysis, available in 30 patients, FDG PET-CT showed low sensitivity (40\%) but higher specificity (73\%) for nodal involvement.

Conclusion FDG PET-CT provides new information in a clinically useful proportion of patients, which leads to changes in treatment strategy, most frequently by detecting previously unidentified metastases, particularly in those with intestinal-type tumours.
\end{abstract}

Keywords Gastric cancer · Cancer staging $\cdot$ PET-CT $\cdot$ Metastases

\section{Introduction}

Gastric adenocarcinoma can have a poor prognosis, especially in Western countries where presentation is often at a more

This article is part of the Topical Collection on Oncology - Digestive tract

Karen D. Bosch

karen.bosch@nhs.net

1 Department of Upper GI Surgery, Guy's \& St Thomas' Hospital, London SE1 7EH, UK

2 Department of Cancer Imaging, School of Biomedical Engineering and Imaging Sciences, King's College London, St Thomas' Hospital, London SE1 7EH, UK

3 King's College London and Guy's \& St Thomas' PET Centre, St Thomas' Hospital, London SE1 7EH, UK advanced stage at the time of diagnosis [1]. Those with early tumours, with no or limited nodal involvement, may have surgical resection combined with either neoadjuvant or adjuvant chemotherapy, whereas patients with metastases (M1) are typically managed by palliative means, including chemotherapy and best supportive care without resection. Therefore, it is important to identify appropriate surgical candidates by enhancing the accuracy of pre-treatment staging, which is the basis for treatment decisions [2]. A further consideration is the potential morbidity and mortality from gastric surgery [3], mandating accurate selection of patients who will benefit from surgery and are not harbouring undetected metastatic disease, which may render surgical intervention futile.

Staging of gastric cancer is typically based on endoscopic biopsy, computed tomography (CT) and laparoscopy for those deemed to have loco-regional disease. Positron emission tomography (PET)-CT combines anatomic and functional 
information to allow diagnosis and staging of cancer. PET-CT with 18-fluoro-2-deoxy-D-glucose (FDG) tracer is commonly used in oncology and has now become more widely available $[4,5]$.

In upper gastrointestinal cancer, FDG PET-CT has been shown to be valuable in patients with oesophageal cancer due to its high diagnostic sensitivity for the primary tumour [6], additional staging information regarding nodal status [7] and detection of distant metastases [8-11] thus identifying those patients with occult metastatic disease, or more advanced locoregional node involvement that would benefit from neo-adjuvant chemotherapy.

The usefulness of FDG PET-CT in preoperative staging of gastric adenocarcinoma in particular has been debated, with wide estimates of published sensitivity for detecting the primary tumour ranging from 60 to $94 \%$ [11-15]. In addition, physiological FDG uptake in the stomach can be variable and a malignant focus of disease may be obscured by physiological or benign pathological uptake in the stomach wall, such as acute gastritis $[16,17]$. Differences in FDG uptake between the histological subtypes have also been reported, with higher uptake in cancers of intestinal type and more advanced tumours [18]. Reports have indicated low rates of detection in more distal tumours, early (T1, T2) tumours [19] and those of diffuse histological subtype [20]. It is therefore not routinely recommended in the UK, according to recent guidelines [21].

As FDG PET-CT has become more routinely available, more studies have examined its role in the staging of gastric adenocarcinoma. Recent reports have estimated the added diagnostic value of FDG PET-CT for detecting occult metastases as 6-10\% [11, 15, 22, 23] with accompanying reductions in surgical morbidity, mortality and cost by the avoidance of unnecessary gastrectomy. Due to relatively low spatial resolution, FDG PET-CT does not add to assessment of primary tumour (T) stage [24]. For the assessment of locoregional lymph nodal staging in gastric cancer, FDG PET-CT is more specific but less sensitive than CT alone [25-27]. However, recent studies have shown poorer prognosis for patients with FDG-avid nodes [11, 28, 29], suggesting an additional role for PET-CT in identifying patients at higher risk of metastasis.

In the present retrospective study, we aimed to investigate the usefulness of PET-CT in the staging work-up of patients with gastric adenocarcinoma and whether this has an impact on subsequent management.

\section{Methods}

\section{Patient population}

The records of patients referred for discussion at the Upper Gastrointestinal Multidisciplinary Team (MDT) meeting at
Guy's and St Thomas' Hospital NHS Foundation Trust between March 2014 and December 2016 were analysed. All patients with a new diagnosis of biopsy-proven gastric adenocarcinoma were identified. Patients with Siewert Type I or II gastro-oesophageal tumours were excluded from the study; Type III tumours were included. We collected demographic data, tumour characteristics, radiographic and FDG PET-CT scan details, and treatment information from the patients' electronic record. TNM stage, as per MDT consensus, was recorded both pre- and post-PET-CT scan. The contemporary 7th Edition of the American Joint Committee on Cancer (AJCC) manual of staging criteria was used to further classify staging information [2]. For those patients who subsequently underwent surgical resection the type of resection, number of lymph nodes resected and number of nodes positive for metastatic adenocarcinoma were recorded.

\section{FDG PET-CT grading}

18-FDG-PET-CT scans were performed using the standard clinical oncology protocol at a single high-volume institution with double reporting as standard procedure. Scans were acquired $90 \mathrm{~min}$ after intravenous injection of $350 \mathrm{MBq}$ FDG (range $283 \mathrm{MBq}$ to $389 \mathrm{MBq}$ ). Images were acquired from skull base to upper thighs with 3 min per bed position using a GE Discovery $710 \mathrm{PET} /$ CT scanner (GE Healthcare, Chicago, USA). A low-dose CT scan $(140 \mathrm{kV}, 10 \mathrm{~mA}, 0.5 \mathrm{~s}$ rotation time, and $40 \mathrm{~mm}$ collimation) was performed at the start of imaging to provide attenuation correction and an anatomical reference. PET images were reconstructed with a time-of-flight ordered subset expectation maximisation algorithm (2 iterations, 24 subsets) with a reconstructed slice thickness of $3.27 \mathrm{~mm}$ and pixel size $4.7 \mathrm{~mm}$. FDG PET-CT scan reports were analysed and grouped as follows:

- 1 = no uptake in primary

- 2 = increased uptake in primary compared to physiological gastric activity only

- 3 = increased uptake in primary and local nodes

- $4=$ increased uptake in primary \pm nodes and distant metastases

Group 4 includes any positivity that would indicate metastatic disease (and therefore lead to M1 staging), which includes both solid organ and distant lymph node disease [2]. In addition, a record was kept of whether FDG uptake unrelated to the gastric primary was described in the PET-CT report, and whether this then led directly to additional investigations. For quantitative analysis, a single, experienced nuclear medicine physician used a dedicated work-station to analyse FDG PET data for the maximum standardised uptake value $\left(\mathrm{SUV}_{\max }\right)$. 


\section{Change in staging following FDG PET-CT}

For each patient who had TNM staging information available both pre- and post-FDG PET-CT, the effect of the additional scan on the staging was grouped as follows:

- $\mathrm{A}=$ negative scan

- $\mathrm{B}=$ no change in stage

- $\mathrm{C}=$ up-stage $\mathrm{N}$

- $\mathrm{D}=$ up-stage $\mathrm{M}$

Group $\mathrm{C}$ indicates local nodes identified and involved on FDG PET-CT (i.e. N0 up-staged to N1, N2 or N3) and group $\mathrm{D}$, newly diagnosed metastases (i.e. M0 up-staged to M1).

\section{Statistics}

To test for statistical differences between mean values, unpaired Student's T test was used. $P$-values below 0.05 were considered statistically significant.

\section{Results}

\section{Patient and tumour characteristics}

A total of 330 patients with newly diagnosed, biopsy-proven gastric adenocarcinoma were identified between March 2014 and December 2016. The majority of patients were male (65\%, ratio 1.9:1) and median age at diagnosis was 73 years (range, 24-95 years). Histological analysis after endoscopic biopsy showed Lauren classifications consisting of $41 \%$ intestinal, $23 \%$ diffuse, $11 \%$ mixed and $24 \%$ not reported. Tumour differentiation at biopsy was $56 \%$ poorly differentiated, $33 \%$ moderately differentiated $2 \%$ well differentiated and $8 \%$ not reported. At the time of initial MDT discussion, following endoscopic biopsy and staging CT, $31 \%$ patients were found to have metastatic disease. These results are summarised in Table 1.

\section{Uptake distribution of FDG}

A total of 105 patients (32\%) underwent PET-CT as part of the staging process. Breakdown of the patient and tumour characteristics of this sub-group of patients is also shown in Table 1. FDG PET-CT scans were not performed in the remainder of cases due to findings of metastatic disease on CT 88 (27\%), frailty and limited treatment decisions $56(17 \%)$, early/straight to surgery $45(14 \%)$, patient declined $2(1 \%)$ and not documented $34(9 \%)$.

Analysis of FDG PET-CT scan reports showed that $86 \%$ of patients had an FDG-avid primary tumour (groups 2-4; 90 of 105 PET-CT scans), with breakdown of distribution as follows: $40(38 \%)$ patients had a positive primary tumour only (group 2), 23 (22\%) had positive primary and locoregional lymph nodes (group 3), and 27 (26\%) had positive primary and distant metastases (group 4) (Fig. 1). Example images are shown in Fig. 2. The average size of FDG negative tumours was $39.7 \mathrm{~mm}$ (range $10-80 \mathrm{~mm}$ ), i.e. tumours were larger than the resolution of PET-CT. The presence of FDG uptake unrelated to the gastric primary was also recorded and found to be present in $71(68 \%)$ of FDG PET-CT scan reports. Thirteen of $105(12 \%)$ patients required further investigation of incidental findings based on FDG PET-CT results. These included endobronchial ultrasound (EBUS) for upper mediastinal nodes $(n=4)$, colonoscopy $(n=2)$, cystoscopy $(n=2)$, fine needle aspiration of supraclavicular node $(n=1)$, breast ultrasound $(n=1)$ and pleural biopsy $(n=1)$. Incidental uptake typically represented distant inflammatory processes. However, in two cases a synchronous primary tumour was newly identified elsewhere (squamous cell carcinoma of the lung and B cell lymphoma of cervical lymph nodes).

Of the 90 FDG positive primary tumours, those of intestinal subtype were more likely to have FDG-avid lymph nodes $(n=13 / 44,30 \%)$ or FDG-avid metastases $(n=15 / 44,34 \%)$ compared with non-intestinal-type tumours $(n=5 / 46,11 \%$ and $n=9 / 46,20 \%$; lymph node and metastasis positivity, respectively; Fig. 3).

\section{FDG-avidity of the primary tumour}

Semi-quantitative analysis of FDG-avidity was obtained by measuring the $\mathrm{SUV}_{\max }$ of the primary tumour. From this analysis, the primary tumour was found to have a mean $\mathrm{SUV}_{\max }$ of $12.0 \pm 0.9$ (mean \pm standard error of the mean (SEM); range, 2.6-41.4). Intestinal-type primary tumours were PET-positive in $93 \%$ (41/44) of cases, compared with 36/46 (78\%) nonintestinal-type tumours (diffuse, mixed). In the remaining 15 cases, histological subtype was not recorded. In addition, mean $\mathrm{SUV}_{\text {max }}$ uptake was significantly higher in tumours of intestinal vs. non-intestinal type tumours (14.1 \pm 1.3 vs. $9.0 \pm$ 0.9 ; mean \pm SEM, $p=0.005)$. Primary tumours that had metastasised also had higher $\mathrm{SUV}_{\max }$ values (11.2 \pm 1.2 for $\mathrm{M} 0$ disease vs. $14.6 \pm 1.7$ for M0; mean $\pm \mathrm{SEM} ; p=0.052$ ).

\section{Changes in staging following FDG PET-CT}

TNM stage prior to and following FDG PET-CT was recorded for each patient where this information was available (105 patients) and the impact of the scan on the staging was grouped (see Methods section, above). New information from FDG PET-CT lead to up-staging in 20/105 patients (groups C and $\mathrm{D} ; 19 \%$ ) by demonstration of previously undetected positive lymph nodes or metastases (Fig. 4). Of those patients who were up-staged, the majority was due to newly detected metastases (group D; 17 patients, 16\%). These newly detected 
Table 1 Overview of patient and tumour characteristics

\begin{tabular}{llll}
\hline Characteristic & & Overall, N $=330, n(\%)$ & PET-CT group, N = 105, $n(\%)$ \\
\hline Sex & Male & $216(65)$ & $74(70)$ \\
Median age & Female & $114(35)$ & $31(30)$ \\
Lauren's classification & Intestinal & $135(41)$ & 69 (range 24-87) \\
& Diffuse & $77(23)$ & $44(42)$ \\
& Mixed & $37(11)$ & $30(29)$ \\
& Other & $3(1)$ & $16(15)$ \\
Differentiation & Not reported & $78(24)$ & $0(0)$ \\
& Well & $8(2)$ & $15(14)$ \\
& Moderate & $110(33)$ & $1(1)$ \\
& Poor & $186(56)$ & $36(34)$ \\
Metastases & Not reported & $26(8)$ & $64(61)$ \\
& Mo & $227(69)$ & $75(4)$ \\
& M1 & $103(31)$ & $30(29)$
\end{tabular}

occult metastases included distant nodes (8 patients), peritoneal nodules (6 patients), liver metastases ( 2 patients) and adrenal metastasis (1 patient). Example images from patients with occult metastases are shown in Fig. 5. In 85 patients (groups $\mathrm{A}$ and $\mathrm{B} ; 81 \%$ ), no new information was provided as the FDG PET-CT was either negative or in agreement with the staging after initial MDT discussion (i.e. staging prior to FDG PET-CT).

\section{Concordance between PET-CT and histological lymph node status}

From a total of 330 patients with newly diagnosed gastric adenocarcinoma, 54 (16\%) underwent resectional surgery. Of these patients, 30 had a pre-surgical FDG PET-CT scan to enable comparison of pre-operative regional lymph node FDG-positivity and histological nodal status. Seventeen patients' nodal status was concordant on FDG PET-CT and histological analysis (Table 2; six FDG and histology positive, 11 FDG and histology negative). Discordance between pre-treatment FDG PET-CT and histological nodal status was seen in 13 patients (Table 2; 4 FDG positive, histology negative, 9 FDG negative, histology positive). Of this discordant group, the majority of those with positive lymph nodes not predicted by PET-CT (i.e. FDG negative, histology positive) were of non-intestinal subtype (78\%). In the concordant groups, the mix of intestinal and non-intestinal histological subtypes were evenly distributed. This suggests that nodal positivity is predicted more accurately in intestinal than nonintestinal tumours. These data correspond to an overall sensitivity and specificity of PET-CT nodal detection of $40 \%$ and $73 \%$, respectively.

Nineteen of 30 patients underwent neo-adjuvant chemotherapy. Of these, discordance between pre-treatment FDG PET-CT and histological nodal status was seen in seven patients (2 FDG positive, histology negative; 5 FDG negative,
Fig. 1 Uptake distribution of FDG $(n=105)$

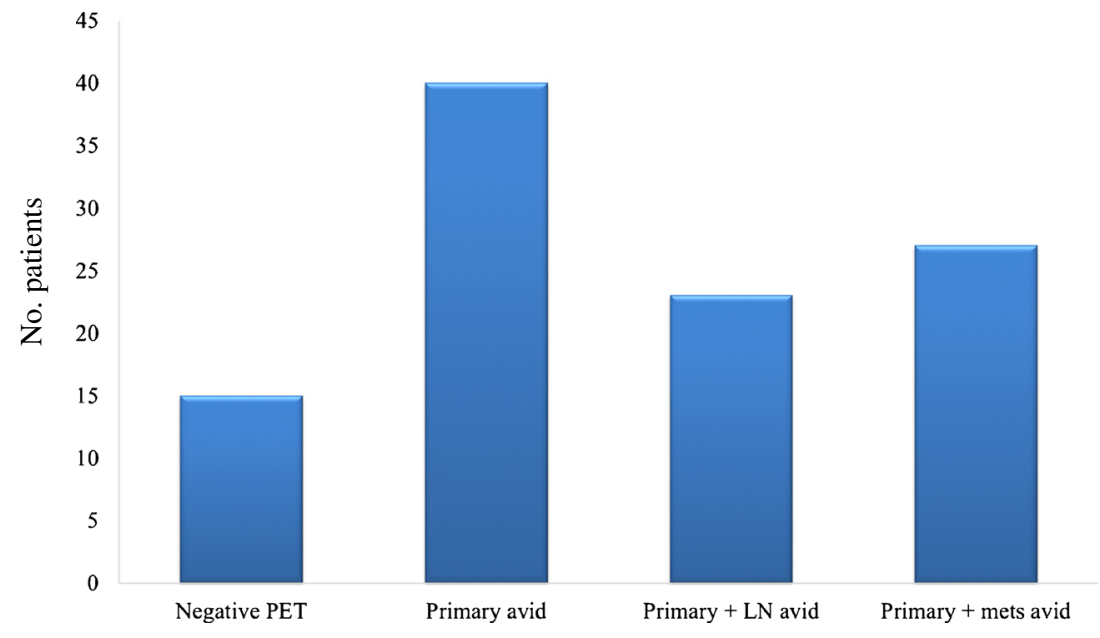


Fig. 2 Example FDG PET-CT images. a Left: maximum intensity projection (MIP) showing diffuse gastric malignancy with multiple left gastric, coeliac, portal and retroperitoneal nodes, bilobar liver metastases and a metastatic superior mediastinal node. Right: axial (PET - top, CT - middle, fused PET-CT -

bottom). Arrows indicate the gastric primary (black), a regional left gastric node (blue), bilobar liver metastases (red), a portal node (green) and a small left superior mediastinal node (orange; left image). b Example FDG PET-CT images from three patients with different uptake distribution.

Shown are axial images, from left to right: MIP, fused PET-CT, CT only; (i) Focal primary tumour uptake, lesser curvature of stomach, (ii) Diffuse primary tumour uptake along both greater and lesser curvatures of stomach, (iii) Lesion along lesser curvature of stomach corresponding to soft tissue on CT. Note photopaenic large simple hepatic cysts a

b

i
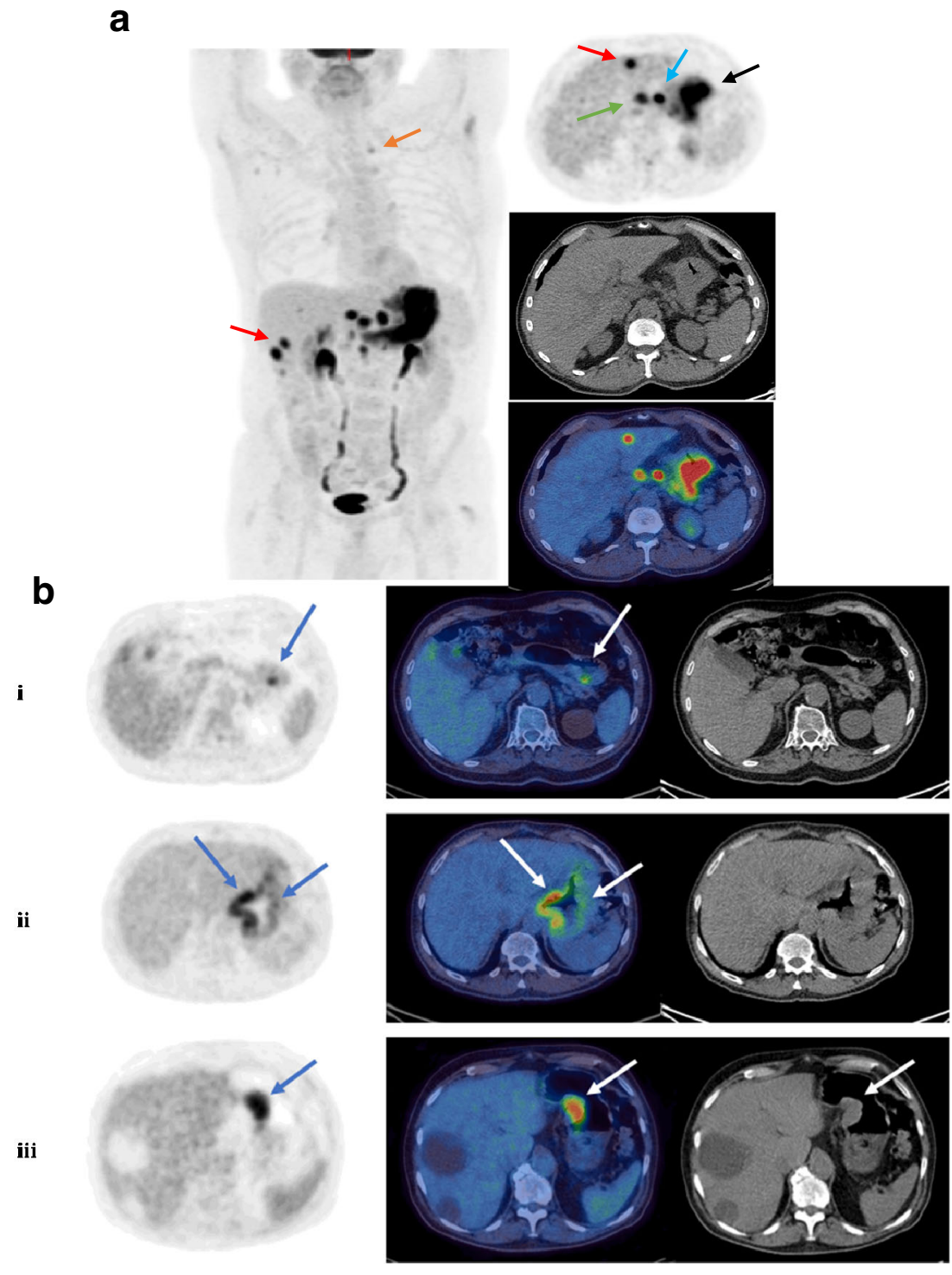

ii

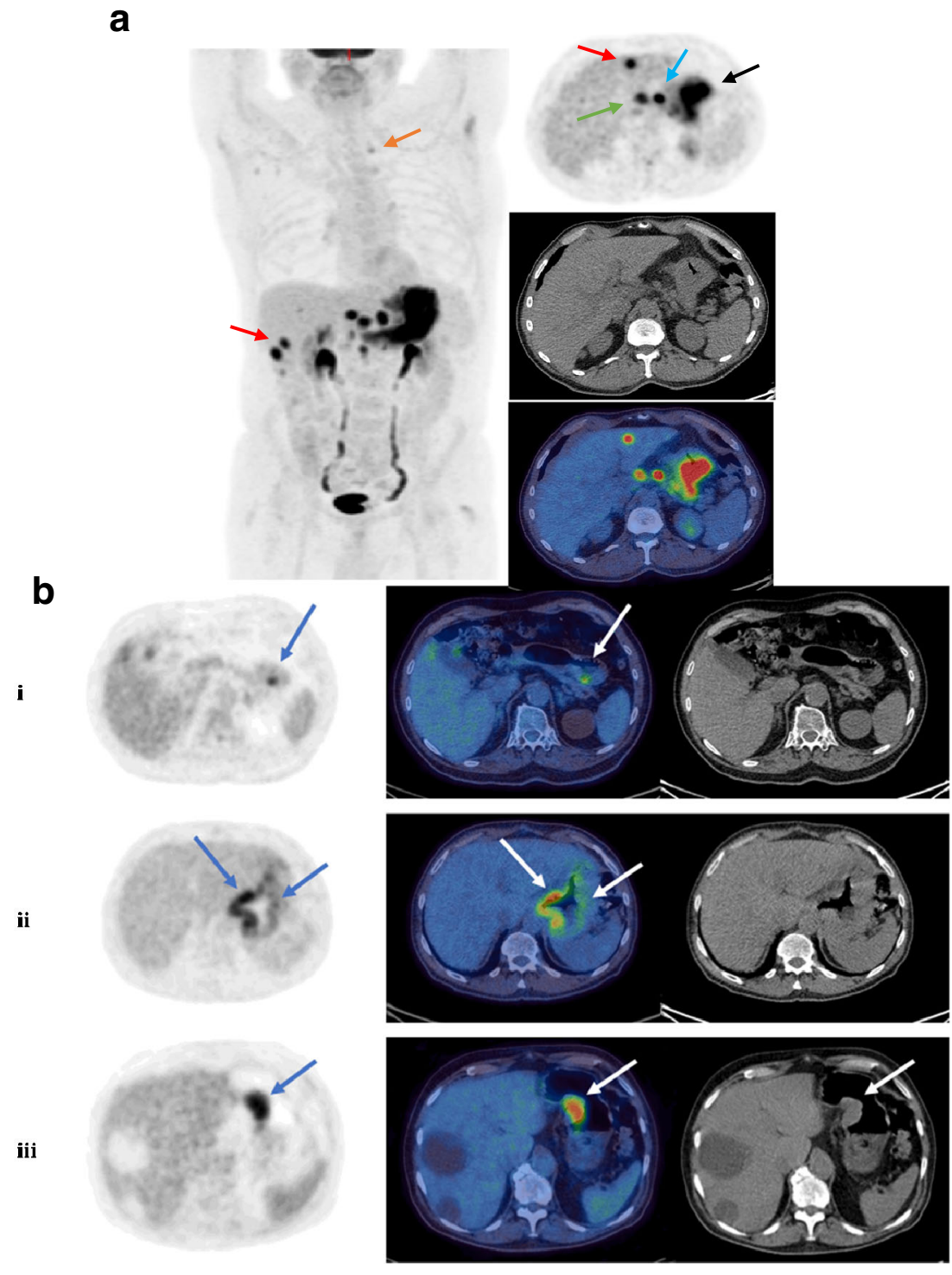

iii
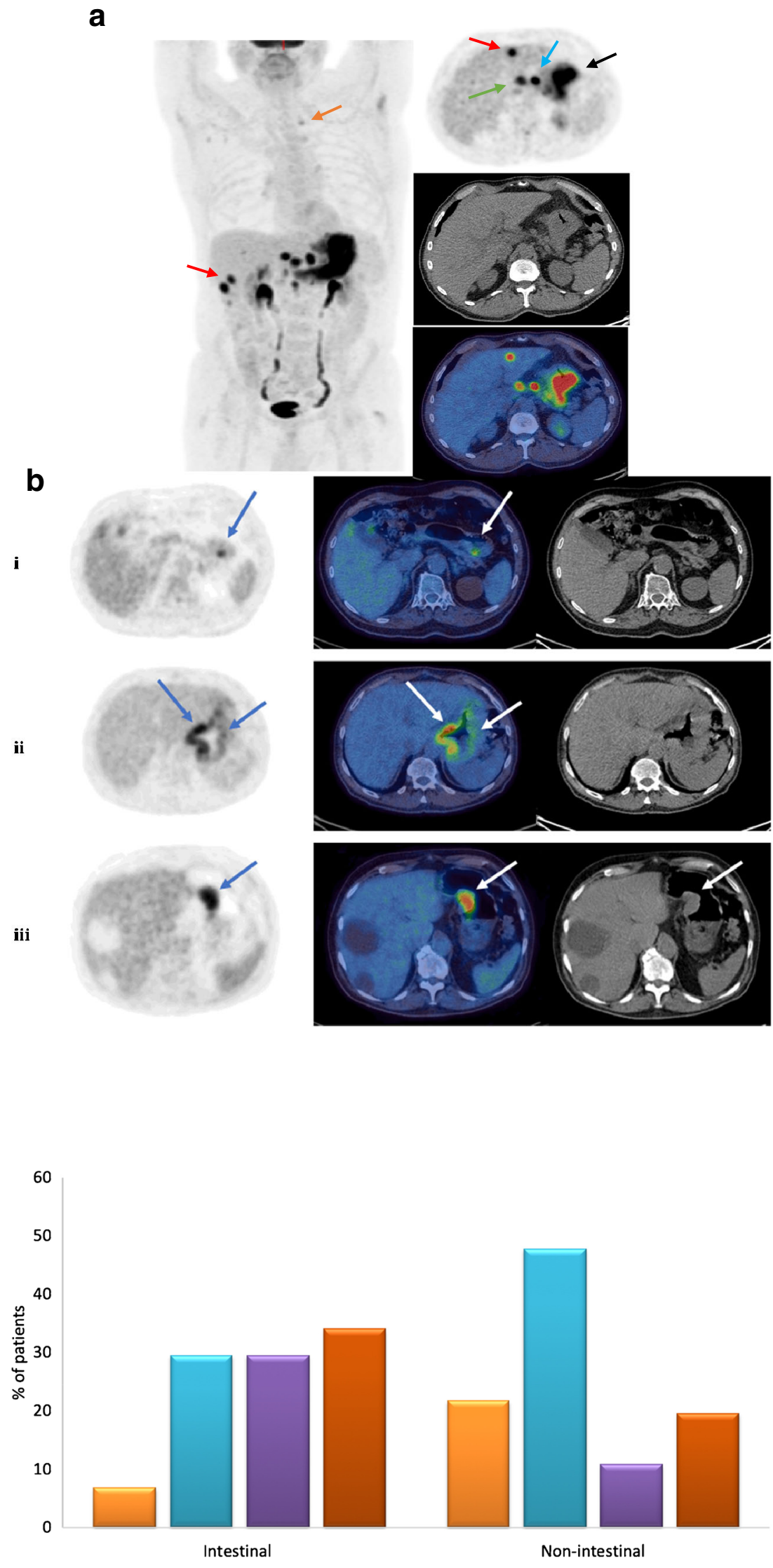

$\square$ Negative PET-CT $\square$ Primary +ve $\square$ Primary + lymph nodes +ve $\square$ Primary + metastases +ve
Fig. 3 Uptake distribution of FDG by histological subtype $(n=$ 90). Percentage of patients for intestinal and non-intestinal subtypes, respectively: negative: FDG PET-CT 7\% (3/44), 22\% $(10 / 46)$; primary +ve: $30 \%(13 /$ 44), $48 \%$ (22/46); primary +ve and lymph nodes +ve: $30 \%$ (13/ $44), 11 \%(5 / 46)$; primary +ve and metastases +ve: $34 \%$ (15/44), $20 \%(9 / 46)$ 
Fig. 4 Change in TNM stage following PET-CT $(n=105)$. Negative PET $=16$ patients; no change $=69$ patients; upstage $\mathrm{N}=3$ patients; upstage $\mathrm{M}=17$ patients

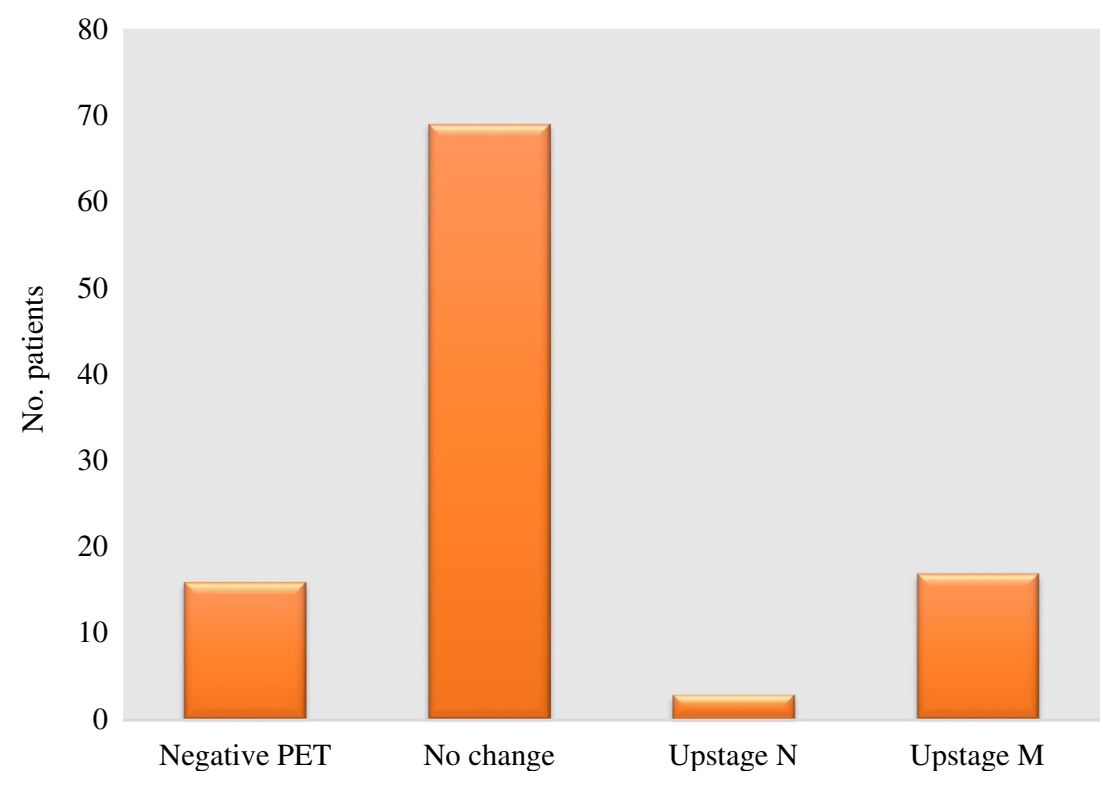

histology positive). Four out of five patients in the neoadjuvant group with positive lymph nodes not predicted by PET-CT were of non-intestinal subtype. Eleven patients proceeded directly to surgery following FDG PET-CT without neo-adjuvant treatment. In two cases, chemotherapy was contraindicated due to gastric outlet obstruction and cardiac comorbidity. In the remaining nine patients, pre-operatively staged as early, six of nine were finally staged as T1/2 (N02) and in three cases T3/4 but N0.

\section{Discussion}

Here we report the results of a large retrospective study evaluating the usefulness of FDG PET-CT in pre-operative staging of gastric cancer. The population is urban and screening programs are not in use. We describe a cohort of predominantly older patients, with twice as many males as females, consistent with previous reports [30,31]. Tumours were mostly poorly differentiated and a substantial proportion (31\%) of patients were found to have metastatic disease at diagnosis.

In order to identify those who are likely to benefit from curative surgery, accurate staging information is essential. The role of FDG PET-CT as part of the staging algorithm in gastric cancer has been debated. This study confirms that it does have a place in modern gastric staging and complements other imaging modalities. We report an additional $16 \%$ of patients in whom occult metastases, consisting of distant lymph nodes or solid organ disease, were identified solely on FDG PET-CT, thus rendering those patients unsuitable a
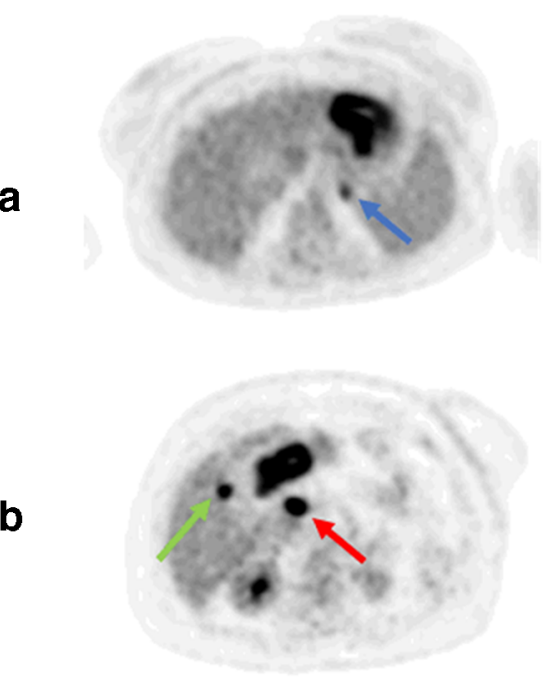

Fig. 5 Example FDG PET-CT images from patients with occult metastases. Shown are axial images, from left to right: MIP, fused PET-CT, CT only; (a) positive gastric primary plus occult adrenal metastasis (blue and
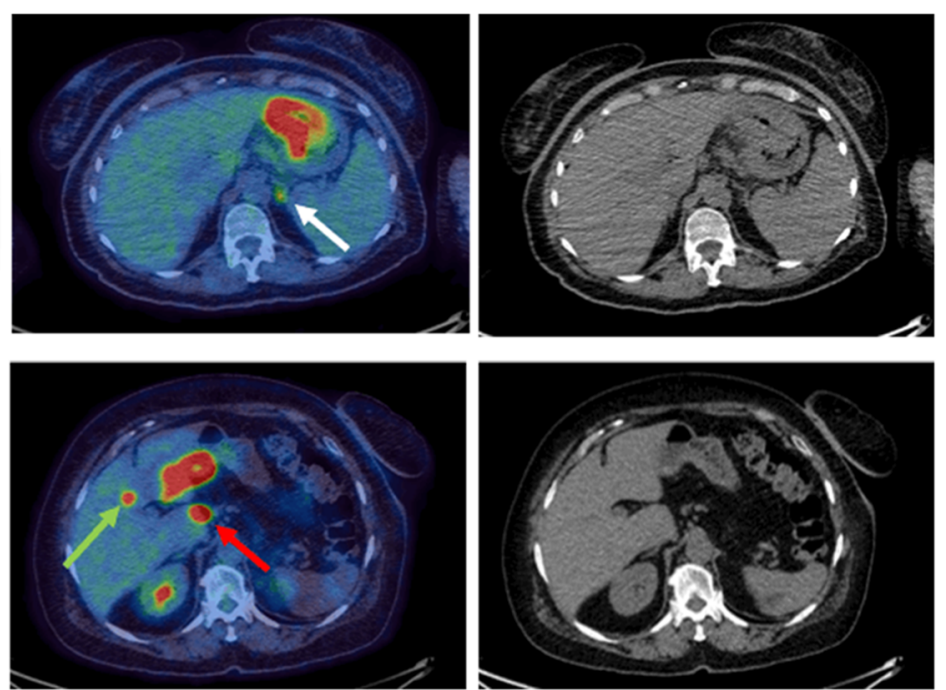

white arrows), (b) positive gastric primary with porta hepatis node (red arrows) plus occult hepatic metastasis (green arrows) 
Table 2 Concordance between lymph node status on FDG PET-CT and histological analysis

\begin{tabular}{lll}
\hline Lymph node status & Histology positive & Histology negative \\
\hline FDG positive & 6 & 4 \\
FDG negative & 9 & 11 \\
\hline
\end{tabular}

for curative surgery. This guides treatment strategies away from the morbidity and mortality associated with a futile surgery, with no benefit for long-term survival, and avoidance of the considerable associated cost. This is consistent with previous studies, which have reported added information regarding occult metastases in up to $10 \%$ of patients $[11,15,22,23]$. A possible reason for the higher detection rate of metastases in our study compared to previous reports include improved scanning technology (e.g. availability of time of flight PET acquisition), resulting in improved sensitivity.

In agreement with previous reports, sensitivity for the primary tumour is greater for the intestinal histological subtype. In addition, we found that tumours of the intestinal type have greater rates of FDG-avid lymph nodal and metastatic positivity. Indeed, of the patients up-staged by new information from FDG PET-CT, more than twice as many were of the intestinal subtype. It is likely that FDG PET-CT is more sensitive to small deposits of intestinal versus non-intestinal-type tumours. This hypothesis is supported by our results, and others, of higher $\mathrm{SUV}_{\max }$ values reflecting this pattern [11], and superior prediction of lymph node metastasis for intestinal tumours when pre-surgical FDG PET-CT is compared with histological analysis. Interestingly, Findlay and colleagues [11] did not detect a difference in the rate of primary tumour avidity between the histological subtypes, despite agreement with our findings on subtype differences in $\mathrm{SUV}_{\max }$.

Lymph nodal status on pre-surgical staging guides oncological and surgical strategy. Several studies have shown limited sensitivity of FDG PET-CT in evaluating lymph node metastases in gastric cancer [32-34], with values for sensitivity typically less than $50 \%$, and the results of the present study are in agreement with these findings. It has been suggested that spatial resolution of FDG PET-CT is not high enough to differentiate the primary tumour from positive perigastric nodes [33], in addition to the frequent finding of nodal metastases in nodes that are not enlarged by CT criteria, i.e. subcentimetre. Our reported specificity in lymph nodal status is much higher than the sensitivity, $73 \%$, although this figure is lower than that reported by others [33, 29]; this was possibly due to patients in our surgical cohort who underwent neoadjuvant chemotherapy and downstaging. Interestingly, recent studies specifically examining the significance of nodal FDG avidity have found poorer prognosis among patients with nodal FDG positivity, suggesting that PET-CT may identify a subset of cancers with a greater propensity to metastasise $[11,29]$.

A number of factors complicating the usefulness of FDG PET-CT in gastric cancer staging have been identified in the present study. One example is that a significant proportion of tumours do not take up the tracer (14\%), so no staging information is added in these patients. This is not a function of smaller tumour size as the average size of $18 \mathrm{~F}-\mathrm{FDG}$ negative tumours is close to $4 \mathrm{~cm}$, with the smallest tumour measuring $1 \mathrm{~cm}$. Additionally, physiological or inflammatory uptake in non-malignant gastric mucosa, for example, induced by Helicobacter pylori infection, can obscure a gastric cancer and provide difficulty with primary tumour identification [35]. Furthermore, more than half of the FDG PET-CT scans reported incidental findings, leading to additional tests and MDT discussion, which resulted in delays to treatment. The incidental findings were usually benign, distant inflammatory processes. However, in two cases a synchronous unrelated primary tumour was identified.

Limitations of the study include its retrospective nature, which means there is a risk that the cohort of patients undergoing additional staging investigations, such as FDG PET-CT, may exhibit a degree of referral bias, potentially overestimating the usefulness of the scans. Comparison of scan and histological results must also be interpreted carefully in view of some patients undergoing neo-adjuvant therapy, therefore potentially changing the histological results and increasing the time interval between staging and histological analysis. Finally, FDG PET-CT scans were initially reported by multiple team members so there is the possibility of interreporter variation. However, this risk is mitigated by the routine practice of double-reporting of scans and a highly experienced team. Additionally, here we reflect real world results rather than controlled experimental conditions.

In conclusion, this study confirms that selected use of FDG PET-CT scanning has a place in the modern staging algorithm of gastric cancer in the UK. Specifically, those patients with no obvious metastases on initial staging, who are eligible for radical curative therapy, would benefit from additional staging information. The most useful aspect is the detection of occult metastases, allowing appropriate identification of surgical candidates and avoiding futile surgery in those with M1 disease. Although valuable information was elucidated in all histological subtypes, FDG PET-CT is particularly sensitive to those tumours of intestinal type. Future, prospective large studies are needed to further investigate the role of FDG PET-CT in N staging and also in the post-treatment phase, such as in the monitoring of response to chemotherapy and prognostic assessment.

Funding The authors acknowledge financial support from the King's College London / University College London Comprehensive Cancer Imaging Centres funded by Cancer Research UK and Engineering and 
Physical Sciences Research Council in association with the Medical Research Council and the Department of Health (C1519/A16463), and the Wellcome Trust EPSRC Centre for Medical Engineering at King's College London (WT203148/Z/16/Z).

\section{Compliance with ethical standards}

Conflict of interest All authors declare no conflict of interest.

Ethical approval We included all patients with gastric adenocarcinoma from MDT records from March 2014 to December 2016 following Institutional Rasearch and Development committee approval. Research Review Board approval was obtained from NRES Committee North West Haydock (REC reference NW / 17 / 0377).

Consent The Committee waived the need for formal consent since anonymised data only would be used for research purposes.

Open Access This article is distributed under the terms of the Creative Commons Attribution 4.0 International License (http:// creativecommons.org/licenses/by/4.0/), which permits unrestricted use, distribution, and reproduction in any medium, provided you give appropriate credit to the original author(s) and the source, provide a link to the Creative Commons license, and indicate if changes were made.

\section{References}

1. Siewert JR, Bottcher K, Stein HJ, Roder JD. Relevant prognostic factors in gastric cancer: ten-year results of the German Gastric Cancer Study. Ann Surg. 1998;228(4):449-61.

2. Washington K. 7th edition of the AJCC cancer staging manual: stomach. Ann Surg Oncol. 2010;17(12):3077-9. https://doi.org/ 10.1245/s10434-010-1362-z.

3. Kim HH, Hyung WJ, Cho GS, Kim MC, Han SU, Kim W, et al. Morbidity and mortality of laparoscopic gastrectomy versus open gastrectomy for gastric cancer: an interim report-a phase III multicenter, prospective, randomized Trial (KLASS Trial). Ann Surg. 2010;251(3):417-20. https://doi.org/10.1097/SLA. 0b013e3181cc8f6b.

4. Juweid ME, Cheson BD. Positron-emission tomography and assessment of cancer therapy. N Engl J Med. 2006;354(5):496-507. https://doi.org/10.1056/NEJMra050276.

5. Scott AM. Current status of positron emission tomography in oncology. Intern Med J. 2001;31(1):27-36.

6. Rasanen JV, Sihvo EI, Knuuti MJ, Minn HR, Luostarinen ME, Laippala P, et al. Prospective analysis of accuracy of positron emission tomography, computed tomography, and endoscopic ultrasonography in staging of adenocarcinoma of the esophagus and the esophagogastric junction. Ann Surg Oncol. 2003;10(8):954-60.

7. Flamen P, Lerut A, Van Cutsem E, De Wever W, Peeters M, Stroobants S, et al. Utility of positron emission tomography for the staging of patients with potentially operable esophageal carcinoma. J Clin Oncol. 2000;18(18):3202-10. https://doi.org/10.1200/ JCO.2000.18.18.3202.

8. Block MI, Patterson GA, Sundaresan RS, Bailey MS, Flanagan FL, Dehdashti F, et al. Improvement in staging of esophageal cancer with the addition of positron emission tomography. Ann Thorac Surg. 1997;64(3):770-6 discussion 6-7.

9. Luketich JD, Friedman DM, Weigel TL, Meehan MA, Keenan RJ, Townsend DW, et al. Evaluation of distant metastases in esophageal cancer: 100 consecutive positron emission tomography scans. Ann Thorac Surg. 1999;68(4):1133-6 discussion 6-7.

10. Blencowe NS, Whistance RN, Strong S, Hotton EJ, Ganesh S, Roach $\mathrm{H}$, et al. Evaluating the role of fluorodeoxyglucose positron emission tomography-computed tomography in multi-disciplinary team recommendations for oesophago-gastric cancer. Br J Cancer. 2013;109(6):1445-50. https://doi.org/10.1038/bjc.2013.478.

11. Findlay JM, Antonowicz S, Segaran A, El Kafsi J, Zhang A, Bradley KM, et al. Routinely staging gastric cancer with (18)FFDG PET-CT detects additional metastases and predicts early recurrence and death after surgery. Eur Radiol. 2019;29(5):2490-8. https://doi.org/10.1007/s00330-018-5904-2.

12. Stahl A, Ott K, Weber WA, Becker K, Link T, Siewert JR, et al. FDG PET imaging of locally advanced gastric carcinomas: correlation with endoscopic and histopathological findings. Eur J Nucl Med Mol Imaging. 2003;30(2):288-95. https://doi.org/10.1007/ s00259-002-1029-5.

13. Yeung HW, Macapinlac H, Karpeh M, Finn RD, Larson SM. Accuracy of FDG-PET in gastric cancer. Preliminary Experience Clin Positron Imaging. 1998;1(4):213-21.

14. Mochiki E, Kuwano H, Katoh H, Asao T, Oriuchi N, Endo K. Evaluation of 18F-2-deoxy-2-fluoro-D-glucose positron emission tomography for gastric cancer. World J Surg. 2004;28(3):247-53. https://doi.org/10.1007/s00268-003-7191-5.

15. Chen J, Cheong JH, Yun MJ, Kim J, Lim JS, Hyung WJ, et al. Improvement in preoperative staging of gastric adenocarcinoma with positron emission tomography. Cancer. 2005;103(11):238390. https://doi.org/10.1002/cncr.21074.

16. Takahashi H, Ukawa K, Ohkawa N, Kato K, Hayashi Y, Yoshimoto K, et al. Significance of (18)F-2-deoxy-2-fluoro-glucose accumulation in the stomach on positron emission tomography. Ann Nucl Med. 2009;23(4):391-7. https://doi.org/10.1007/s12149-0090255-3.

17. Koga H, Sasaki M, Kuwabara Y, Hiraka K, Nakagawa M, Abe K, et al. An analysis of the physiological FDG uptake pattern in the stomach. Ann Nucl Med. 2003;17(8):733-8.

18. Mukai K, Ishida Y, Okajima K, Isozaki H, Morimoto T, Nishiyama S. Usefulness of preoperative FDG-PET for detection of gastric cancer. Gastric Cancer. 2006;9(3):192-6. https://doi.org/10.1007/ s10120-006-0374-7.

19. Dassen AE, Lips DJ, Hoekstra CJ, Pruijt JF, Bosscha K. FDG-PET has no definite role in preoperative imaging in gastric cancer. Eur $\mathrm{J}$ Surg Oncol. 2009;35(5):449-55. https://doi.org/10.1016/j.ejso. 2008.11.010.

20. Berger KL, Nicholson SA, Dehdashti F, Siegel BA. FDG PET evaluation of mucinous neoplasms: correlation of FDG uptake with histopathologic features. AJR Am J Roentgenol. 2000;174(4): 1005-8. https://doi.org/10.2214/ajr.174.4.1741005.

21. Smyth EC, Verheij M, Allum W, Cunningham D, Cervantes A, Arnold D, et al. Gastric cancer: ESMO Clinical Practice Guidelines for diagnosis, treatment and follow-up. Ann Oncol. 2016;27(suppl 5):v38-49. https://doi.org/10.1093/annonc/ mdw350.

22. Serrano OK, Love C, Goldman I, Huang K, $\mathrm{Ng} \mathrm{N}$, Abraham T, et al. The value of FDG-PET in the staging of gastric adenocarcinoma: A single institution retrospective review. J Surg Oncol. 2016;113(6): 640-6. https://doi.org/10.1002/jso.24190.

23. Smyth E, Schoder H, Strong VE, Capanu M, Kelsen DP, Coit DG, et al. A prospective evaluation of the utility of 2-deoxy-2-[(18) F]fluoro-D-glucose positron emission tomography and computed tomography in staging locally advanced gastric cancer. Cancer. 2012;118(22):5481-8. https://doi.org/10.1002/cncr.27550.

24. Kitajima K, Nakajo M, Kaida H, Minamimoto R, Hirata K, Tsurusaki M, et al. Present and future roles of FDG-PET/CT imaging in the management of gastrointestinal cancer: an update. 
Nagoya J Med Sci. 2017;79(4):527-43. https://doi.org/10.18999/ nagjms.79.4.527.

25. Gauthe M, Richard-Molard M, Cacheux W, Michel P, Jouve JL, Mitry E, et al. Role of fluorine 18 fluorodeoxyglucose positron emission tomography/computed tomography in gastrointestinal cancers. Dig Liver Dis. 2015;47(6):443-54. https://doi.org/10. 1016/j.dld.2015.02.005.

26. Malibari N, Hickeson M, Lisbona R. PET/computed tomography in the diagnosis and staging of gastric cancers. PET Clin. 2015;10(3): 311-26. https://doi.org/10.1016/j.cpet.2015.03.008.

27. Kawanaka Y, Kitajima K, Fukushima K, Mouri M, Doi H, Oshima T, et al. Added value of pretreatment (18)F-FDG PET/CT for staging of advanced gastric cancer: Comparison with contrast-enhanced MDCT. Eur J Radiol. 2016;85(5):989-95. https://doi.org/10.1016/j. ejrad.2016.03.003.

28. Kwon HW, An L, Kwon HR, Park S, Kim S. Preoperative nodal (18)F-FDG avidity rather than primary tumor avidity determines the prognosis of patients with advanced gastric cancer. J Gastric Cancer. 2018;18(3):218-29. https://doi.org/10.5230/jgc.2018.18. e23.

29. Kudou M, Kosuga T, Kubota T, Okamoto K, Komaksu S, Shoda K, et al. Value of preoperative PET-CT in the prediction of pathological stage of gastric cancer. Ann Surg Oncol. 2018;25(6):1633-9. https://doi.org/10.1245/s10434-018-6455-0.

30. Anderson WF, Camargo MC, Fraumeni JF Jr, Correa P, Rosenberg PS, Rabkin CS. Age-specific trends in incidence of noncardia gastric cancer in US adults. JAMA. 2010;303(17):1723-8. https:// doi.org/10.1001/jama.2010.496.

31. Jemal A, Bray F, Center MM, Ferlay J, Ward E, Forman D. Global cancer statistics. CA Cancer J Clin. 2011;61(2):69-90. https://doi. org/10.3322/caac.20107.

32. Kim EY, Lee WJ, Choi D, Lee SJ, Choi JY, Kim BT, et al. The value of PET/CT for preoperative staging of advanced gastric cancer: comparison with contrast-enhanced CT. Eur J Radiol. 2011;79(2): 183-8. https://doi.org/10.1016/j.ejrad.2010.02.005.

33. Yun M. Imaging of gastric cancer metabolism using 18 F-FDG PET/CT. J Gastric Cancer. 2014;14(1):1-6. https://doi.org/10. 5230/jgc.2014.14.1.1.

34. Yang QM, Kawamura T, Itoh H, Bando E, Nemoto M, Akamoto S, et al. Is PET-CT suitable for predicting lymph node status for gastric cancer? Hepatogastroenterology. 2008;55(82-83):782-5.

35. Kobayashi S, Ogura M, Suzawa N, Horiki N, Katsurahara M, Ogura T, et al. (18)F-FDG uptake in the stomach on screening PET/CT: value for predicting Helicobacter pylori infection and chronic atrophic gastritis. BMC Med Imaging. 2016;16(1):58. https://doi.org/10.1186/s12880-016-0161-9.

Publisher's note Springer Nature remains neutral with regard to jurisdictional claims in published maps and institutional affiliations. 